\title{
Inflation in a viscous fluid model
}

\author{
Kazuharu Bamba ${ }^{1, a}$, Sergei D. Odintsov ${ }^{2,3}$ \\ ${ }^{1}$ Division of Human Support System, Faculty of Symbiotic Systems Science, Fukushima University, Fukushima 960-1296, Japan \\ ${ }^{2}$ Institut de Ciencies de lEspai (IEEC-CSIC), Campus UAB, Carrer de Can Magrans, Cerdanyola del Valles, 08193 Barcelona, Spain \\ ${ }^{3}$ Institució Catalana de Recerca i Estudis Avançats (ICREA), Passeig Lluís Companys, 2308010 Barcelona, Spain
}

Received: 14 October 2015 / Accepted: 20 December 2015 / Published online: 18 January 2016

(c) The Author(s) 2016. This article is published with open access at Springerlink.com

\begin{abstract}
We explore a fluid description of the inflationary universe. In particular, we investigate a fluid model in which the equation of state (EoS) for a fluid includes bulk viscosity. We find that the three observables of inflationary cosmology, i.e., the spectral index of the curvature perturbations, the tensor-to-scalar ratio of the density perturbations, and the running of the spectral index, can be consistent with the recent Planck results. We also reconstruct the explicit EoS for a fluid from the spectral index of the curvature perturbations compatible with the Planck analysis. In the reconstructed models of a fluid, the tensor-to-scalar ratio of the density perturbations can satisfy the constraints obtained from the Planck satellite. The running of the spectral index can explain the Planck data. In addition, it is demonstrated that in the reconstructed models of a fluid, the graceful exit from inflation can be realized. Moreover, we show that the singular inflation can occur in a fluid model. Furthermore, we show that a fluid description of inflation can be equivalent to the description of inflation in terms of scalar field theories.
\end{abstract}

\section{Introduction}

The various precise properties on inflation in the early universe [1-5] has been revealed by the recent cosmological observations on the anisotropy of the cosmic microwave background (CMB) radiation such as the Planck satellite [6,7] and the BICEP2 experiment [8,9], in addition to the Wilkinson Microwave anisotropy probe (WMAP) $[10,11]$. The nature of inflation can be known from the spectrum of the primordial density perturbations [12-14].

Recently, in Refs. $[15,16]$, the convenient fluid description of the inflationary universe has been proposed in terms of scalar field theories, fluid models [17], and $F(R)$ gravity theories [18-26]. Especially, the three observables of inflationary models, i.e., the spectral index of the curvature per-

a e-mail: bamba@sss.fukushima-u.ac.jp turbations, the tensor-to-scalar ratio of the density perturbations, and the running of the spectral index, have been represented by using quantities in scalar field theories, fluid models, and $F(R)$ gravity theories. Fluid models have been applied to cosmological issues such as inflation [27-33] and dark energy [34] (for recent reviews, see [17,35]).

In this paper, by extending the preceding investigations of a fluid description [16] for the inflationary universe, we derive the slow-roll conditions and construct the formulas of the observables for inflationary models of the spectral index $n_{\mathrm{s}}$ of the curvature perturbations, the tensor-to-scalar ratio $r$ of the density perturbations, and the running $\alpha_{\mathrm{S}}$ of the spectral index. We analyze the equation of state (EoS) for a fluid including bulk viscosity and examine a fluid model in which $n_{\mathrm{s}}, r$, and $\alpha_{\mathrm{s}}$ can be compatible with the recent Planck results.

In addition, we explicitly reconstruct the EoS for a fluid from the spectral index $n_{\mathrm{s}}$ of the curvature perturbations. Particularly, we use the expression of $n_{\mathrm{S}}$ as a function of the number of $e$-folds $N$ during inflation in the inflationary models including the Starobinsky inflation [5], from which the value of $n_{\mathrm{s}}$, consistent with the recent Planck analysis, can be obtained. This reconstruction method for scalar field theories has been proposed in Ref. [36]. In this work, we present the reconstruction procedure in a fluid description. We also show that in the reconstructed models of a fluid, the slow-roll inflation, i.e., the de Sitter inflation, can occur. In these fluid models, the tensor-to-scalar ratio $r$ of the density perturbations can meet the constraints acquired by the Planck satellite. The running $\alpha_{\mathrm{S}}$ of the spectral index can explain the Planck results. Moreover, we verify that in the reconstructed models of a fluid, the graceful exit from inflation can be realized. Furthermore, we demonstrate that in the fluid models, the singular inflation [37-40] can occur. In addition, we explain the equivalence between a fluid description of inflation and the description of inflation in terms of scalar field theories. We use units of $k_{\mathrm{B}}=c=\hbar=1$ and express the gravitational 
constant $8 \pi G_{\mathrm{N}}$ by $\kappa^{2} \equiv 8 \pi / M_{\mathrm{Pl}}^{2}$ with the Planck mass of $M_{\mathrm{Pl}}=G_{\mathrm{N}}^{-1 / 2}=1.2 \times 10^{19} \mathrm{GeV}$.

The organization of the paper is the following. In Sect. 2, we explain a fluid description of the inflationary universe. In particular, we present a fluid model in which the EoS for a fluid includes bulk viscosity and show that the fluid model can explain the recent Planck results of the three observables for inflationary models. In Sect. 3, we reconstruct the EoS for a fluid from the spectral index of the curvature perturbations. We certify that inflation can happen in the reconstructed models of a fluid, and that the tensor-to-scalar ratio of the density perturbations can be consistent with the Planck analysis. In Sect. 4, we investigate that the graceful exit from inflation can be realized in the fluid models reconstructed above. In Sect. 5, we consider the singular inflation in a fluid model. In Sect. 6, we show that a fluid description of inflation can be equivalent to the description of inflation in terms of scalar field theories. Conclusions are presented in Sect. 7. In Appendix A, the slow-roll parameters in a fluid description are given.

\section{Fluid description of inflation}

We consider the case that the so-called slow-roll inflation driven by the potential $V(\phi)$ of a scalar field $\phi$ occurs, which plays a roll of the inflaton field. We explain the procedure [15] to represent the slow-roll parameters in terms of the Hubble parameter and its derivatives of the number of $e$-folds during inflation. Furthermore, with these representations of the slow-roll parameters, we describe the observables of inflationary models, namely, the spectral index of the curvature perturbations, the tensor-to-scalar ratio of the density perturbations, and the running of the spectral index in fluid models [16].

\subsection{Slow-roll parameters}

The action of $\phi$ with the Einstein-Hilbert term is given by

$S=\int \mathrm{d}^{4} x \sqrt{-g}\left(\frac{R}{2 \kappa^{2}}-\frac{1}{2} \partial_{\mu} \phi \partial^{\mu} \phi-V(\phi)\right)$.

Here, $g$ is the determinant of the metric $g_{\mu \nu}$ and $R$ is the scalar curvature. For the slow-roll inflation, the spectral index $n_{\mathrm{S}}$ of the curvature perturbations (i.e., the scalar mode of the density perturbations), the tensor-to-scalar ratio $r$ of the density perturbations, and the running of the spectral index $\alpha_{\mathrm{s}} \equiv \mathrm{d} n_{\mathrm{s}} / \mathrm{d} \ln k$, where $k$ is the absolute value of the wave number $\boldsymbol{k}$, are written as

$n_{\mathrm{s}}-1=-6 \epsilon+2 \eta, \quad r=16 \epsilon, \quad \alpha_{\mathrm{s}}=16 \epsilon \eta-24 \epsilon^{2}-2 \xi^{2}$, where $\epsilon, \eta$, and $\xi$ are the slow-roll parameters, defined as

$$
\begin{aligned}
\epsilon & \equiv \frac{1}{2 \kappa^{2}}\left(\frac{V^{\prime}(\phi)}{V(\phi)}\right)^{2}, \quad \eta \equiv \frac{1}{\kappa^{2}} \frac{V^{\prime \prime}(\phi)}{V(\phi)}, \\
\xi^{2} & \equiv \frac{1}{\kappa^{4}} \frac{V^{\prime}(\phi) V^{\prime \prime \prime}(\phi)}{(V(\phi))^{2}} .
\end{aligned}
$$

Here, the prime shows the derivative with respect to $\phi$ as $V^{\prime}(\phi) \equiv \mathrm{d} V(\phi) / \mathrm{d} \phi$. Throughout this paper, the prime denotes the derivative with respect to the argument of the function, to which the prime operates.

We take the flat Friedmann-Lemaître-Robertson-Walker $\left(\right.$ FLRW) metric $\mathrm{d} s^{2}=-\mathrm{d} t^{2}+a^{2}(t) \sum_{i=1,2,3}\left(\mathrm{~d} x^{i}\right)^{2}$, where $a(t)$ is the scale factor. The Hubble parameter is defined by $H \equiv \dot{a} / a$, where the dot means the time derivative.

We express the slow-roll parameters in terms of $H$, which can be represented as $H=H(N)$, namely, as a function of the number of $e$-folds $N$ during inflation, defined as $N \equiv$ $\ln \left(a_{\mathrm{f}} / a_{\mathrm{i}}\right)=\int_{t_{\mathrm{i}}}^{t_{\mathrm{f}}} H \mathrm{~d} t$, where $a_{\mathrm{i}}$ and $a_{\mathrm{f}}$ are the values of the scale factor $a$ at the initial time $t_{\mathrm{i}}$ and the end time $t_{\mathrm{f}}$ of inflation, respectively. To execute this task, with a new scalar field $\varphi$, we redefine $\phi$ as $\phi=\phi(\varphi)$, where $\varphi$ is identified with $N$. We introduce a positive quantity $\omega(\varphi)(>0)$ defined as $\omega(\varphi) \equiv(\mathrm{d} \phi / \mathrm{d} \varphi)^{2}$, and represent $V$ as a function of $\varphi$, i.e., $V(\varphi) \equiv V(\phi(\varphi))$. In the FLRW background, we derive the gravitational equations and rewrite them by using $\omega(\varphi)$ and $V(\varphi)$. By solving the gravitational equations with respect to $\omega(\varphi)$ and $V(\varphi)$, we obtain [15]

$$
\begin{aligned}
& \omega(\varphi)=-\left.\frac{2}{\kappa^{2}} \frac{H^{\prime}(N)}{H(N)}\right|_{N=\varphi}, \\
& V(\varphi)=\left.\frac{1}{\kappa^{2}}(H(N))^{2}\left(3+\frac{H^{\prime}(N)}{H(N)}\right)\right|_{N=\varphi},
\end{aligned}
$$

with $H^{\prime}(N) \equiv \mathrm{d} H(N) / \mathrm{d} N$. Here, the representations of $H=H(N)$ and $\varphi=N$ are acquired as solutions for the equation of motion of $\phi$ or $\varphi$, and the gravitational field equations. It is seen from the first equation in (2.4) that since $\omega(\varphi)>0$, we have $H^{\prime}(N)<0$. The slow-roll parameters in (2.3) can be rewritten by using $\omega(\varphi)$ and $V(\varphi)$. Accordingly, through the expressions of $\omega(\varphi)$ and $V(\varphi)$ in (2.4), the slow-roll parameters can be described in terms of $H(N)$ and its derivatives with respect to $N$. The resultant expressions have been given in Ref. [15].

\subsection{Representation of a fluid}

For a general fluid model, the EoS is given by

$P(N)=-\rho(N)+f(\rho)$,

where $\rho$ is the energy density of a fluid, $P$ is its pressure, and $f(\rho)$ is an arbitrary function of $\rho$. In the flat FLRW background, for such a fluid model, the gravitational field 
equations read

$$
\begin{aligned}
\frac{3}{\kappa^{2}}(H(N))^{2} & =\rho, \\
-\frac{2}{\kappa^{2}} H(N) H^{\prime}(N) & =\rho+P,
\end{aligned}
$$

Since the EoS can be expressed as $\rho(N)+P(N)=$ $f(\rho)$, the second gravitational equation is rewritten to $-\left(2 / \kappa^{2}\right) H(N) H^{\prime}(N)=f(\rho)$. Similarly, with the expression of the EoS shown above, the conservation law $0=$ $\rho^{\prime}(N)+3(\rho(N)+P(N))$ becomes $0=\rho^{\prime}(N)+3 f(\rho)$, where $\rho^{\prime}(N) \equiv \mathrm{d} \rho(N) / \mathrm{d} N$. From these second gravitational equation and conservation law, we acquire

$$
\frac{2}{\kappa^{2}}(H(N))^{2}\left[\left(\frac{H^{\prime}(N)}{H(N)}\right)^{2}+\frac{H^{\prime \prime}(N)}{H(N)}\right]=3 f^{\prime}(\rho) f(\rho),
$$

with $f^{\prime}(\rho) \equiv \mathrm{d} f(\rho) / \mathrm{d} \rho$. Owing to this equation, it is possible to express $H(N)$ and its derivatives with respect to $N$ only with $\rho(N)$ and $f(\rho(N))$. Therefore, the slow-roll parameters can be described in terms of $\rho(N)$ and $f(\rho(N))$, as is presented in Appendix A. As a result, by substituting the representations of the slow-roll parameters in Appendix A into the expressions of observables of the inflationary models in (2.3), we obtain the fluid description of $n_{\mathrm{s}}, r$, and $\alpha_{\mathrm{s}}$. In Ref. [16], the explicit expressions of $n_{\mathrm{s}}, r$, and $\alpha_{\mathrm{s}}$ are shown. ${ }^{1}$

The EoS for a fluid can also be represented as $w(N) \equiv$ $P(N) / \rho(N)=-1+f(\rho) / \rho(N)$, from which we find $f(\rho) / \rho(N)=w(N)+1$. When $|f(\rho) / \rho(N)| \ll 1$, and $f(\rho)$ and $\rho$ vary very slowly in the inflationary stage, the approximate expressions of $n_{\mathrm{s}}, r$, and $\alpha_{\mathrm{s}}$ read [16]

$$
\begin{aligned}
\left(n_{\mathrm{s}}, r, \alpha_{\mathrm{s}}\right) \approx & \left(1-6 \frac{f(\rho)}{\rho(N)}, 24 \frac{f(\rho)}{\rho(N)},-9\left(\frac{f(\rho)}{\rho(N)}\right)^{2}\right) \\
= & (1-6(w(N)+1), 24(w(N)+1), \\
& \left.-9(w(N)+1)^{2}\right),
\end{aligned}
$$

where in deriving (2.10), we have used the relation $f(\rho) / \rho(N)=w(N)+1$.

2.3 Fluid model in which the EoS for a fluid includes bulk viscosity

We investigate a fluid with the following EoS:

$P=-\rho+A \rho^{\beta}+\zeta(H)$

where $A$ and $\beta$ are constants, and $\zeta(H)$ is the bulk viscosity. As a specific case, we consider $\zeta(H)$ to have the following form:

\footnotetext{
1 The other way to describe $\alpha_{\mathrm{s}}$ has been examined in Ref. [41].
}

$\zeta(H)=\bar{\zeta} H^{\gamma}$

where $\bar{\zeta}$ and $\gamma$ are constants. We note that the mass dimension of $A$ is $-4(\beta-1)$, whereas that of $\bar{\zeta}$ is $-(\gamma-4)$. From the Friedmann Eq. (2.6) for the expanding universe $(H>0)$, we get $H=(\kappa / \sqrt{3}) \sqrt{\rho}$. Hence, $\zeta(H)$ can be written as a function of $\rho$, namely, $\zeta(H)=\zeta(H(\rho))$. Consequently, by comparing Eq. (2.5) with Eq. (2.11) and using Eq. (2.12), we acquire

$f(\rho)=A \rho^{\beta}+\zeta(H(\rho))=A \rho^{\beta}+\bar{\zeta}\left(\frac{\kappa}{\sqrt{3}}\right)^{\gamma} \rho^{\gamma / 2}$.

Here, we state a physical reason why we have considered the case that $\zeta(H)$ is expressed by a power in $H$ as given in Eq. (2.12) and hence $f(\rho)$ is represented by the linear combination of two kinds of a power in $\rho$ as shown in Eq. (2.13). It is assumed that only for such a case $f(\rho)$ is expressed by a series of a power in $\rho$, and through a phenomenological approach, it is possible to analytically study the quantitative features of the EoS for a fluid to realize inflation in which the three observables of inflationary models, namely, the spectral index of the curvature perturbations, the tensor-to-scalar ratio of the density perturbations, and the running of the spectral index, can explain the recent Planck results, as is demonstrated below.

The Planck analysis $[6,7]$ has shown that $n_{\mathrm{s}}=0.968 \pm$ 0.006 (68\% CL), $r<0.11(95 \% \mathrm{CL})$, and $\alpha_{\mathrm{s}}=-0.003 \pm$ $0.007(68 \% \mathrm{CL})$. If $f(\rho) / \rho(N)=4.35 \times 10^{-3}$, i.e., $w=$ -0.996 , from Eq. (2.9) or (2.10), we have $\left(n_{\mathrm{s}}, r, \alpha_{\mathrm{s}}\right)=$ $\left(0.974,0.104,-1.70 \times 10^{-4}\right)$. These results are consistent with the Planck data.

We here demonstrate that it is possible to realize these Planck results by choosing appropriate values of the model parameters in Eq. (2.11) with Eq. (2.12). In other words, we explicitly derive the values of the model parameters leading to $f(\rho) / \rho=4.35 \times 10^{-3}$. It follows from Eq. (2.13) that

$$
\begin{aligned}
\frac{f(\rho)}{\rho}= & A \rho_{\mathrm{c}}^{\beta-1}\left(\frac{\rho}{\rho_{\mathrm{c}}}\right)^{\beta-1}+\bar{\zeta}\left(\frac{\kappa}{\sqrt{3}}\right)^{\gamma} \\
& \times \rho_{\mathrm{c}}^{\gamma / 2-1}\left(\frac{\rho}{\rho_{\mathrm{c}}}\right)^{\gamma / 2-1} \\
= & A \rho_{\mathrm{c}}^{\beta-1}\left(\frac{H_{\mathrm{inf}}}{H_{0}}\right)^{2(\beta-1)}+\bar{\zeta}\left(\frac{\kappa}{\sqrt{3}}\right)^{\gamma} \\
& \times \rho_{\mathrm{c}}^{\gamma / 2-1}\left(\frac{H_{\mathrm{inf}}}{H_{0}}\right)^{\gamma-2},
\end{aligned}
$$

where $\rho_{\mathrm{c}} \equiv 3 H_{0}^{2} / \kappa^{2}=8.10 \times 10^{-47} \mathrm{GeV}^{4}$ is the critical density, $H_{0}=100 h \mathrm{~km} \mathrm{sec}^{-1} \mathrm{Mpc}^{-1}=2.13 h \times 10^{-42} \mathrm{GeV}$ with $h=0.678$ [6] is the current Hubble parameter [42], and $H_{\text {inf }}$ is the Hubble parameter at the inflationary stage. For 
simplicity, we set $\gamma=2 \beta$. In this case, from Eq. (2.15), we obtain

$$
\begin{aligned}
\frac{f(\rho)}{\rho} & =J\left(\frac{H_{\mathrm{inf}}}{H_{0}}\right)^{2(\beta-1)}, \\
J & \equiv\left[A+\bar{\zeta}\left(\frac{\kappa}{\sqrt{3}}\right)^{2 \beta}\right] \rho_{\mathrm{c}}^{\beta-1} .
\end{aligned}
$$

For the simplest case that $\beta=1$, when $J=4.35 \times$ $10^{-3}$, regardless of the scale of inflation $H_{\text {inf }}$, the Planck results can be realized. Moreover, in the case that $\beta=2$, for example, if $\left(H_{\text {inf }}, J\right)=\left(1.0 \times 10^{10} \mathrm{GeV}, 9.10 \times 10^{-107}\right)$, $\left(1.0 \times 10^{5} \mathrm{GeV}, 9.10 \times 10^{-97}\right)$, we can explain the Planck data.

\section{Reconstruction of the EoS for a fluid from the spectral index}

In this section, we reconstruct the EoS for a fluid from the spectral index of the curvature perturbations. Such a reconstruction has been studied for the case of scalar field theories in Ref. [36].

\subsection{Reconstruction procedure in a fluid description}

For the slow-roll inflation in scalar field theories, whose action is given by Eq. (2.1), the spectral index $n_{\mathrm{s}}$ of the curvature perturbations, the tensor-to-scalar ratio $r$ of the density perturbations, and the running $\alpha_{\mathrm{s}}$ of the spectral index are derived as follows [36]:

$$
\begin{aligned}
n_{\mathrm{s}}-1 & =\frac{\mathrm{d}}{\mathrm{d} N}\left[\ln \left(\frac{1}{V^{2}(N)} \frac{\mathrm{d} V(N)}{\mathrm{d} N}\right)\right], \\
r & =\frac{8}{V(N)} \frac{\mathrm{d} V(N)}{\mathrm{d} N}, \\
\alpha_{\mathrm{s}} & =-\frac{\mathrm{d}^{2}}{\mathrm{~d} N^{2}}\left[\ln \left(\frac{1}{V^{2}(N)} \frac{\mathrm{d} V(N)}{\mathrm{d} N}\right)\right] .
\end{aligned}
$$

Similarly to the case of scalar field theories, in a fluid model, it is possible to reconstruct the EoS for a fluid from the spectral index $n_{\mathrm{s}}$ of the curvature perturbations. If we have the form of $n_{\mathrm{s}}$ as a function of $N$, by using the first relation in (3.1), we can obtain the expression of $V(N)$. Thanks to the Friedmann Eq. (2.6), the Hubble parameter is related to $V(N)$, and hence we get $H=H(N)$. In a fluid model, with the other gravitational field Eq. (2.7), we can acquire the form of $f(\rho)$ through the EoS in Eq. (2.5).

\subsection{Inflationary models with $n_{\mathrm{s}}-1=-2 / N$}

We demonstrate the reconstruction procedure in a fluid description by exploring the inflationary models in which $n_{\mathrm{s}}$ is given by
$n_{\mathrm{s}}-1=-\frac{2}{N}$

It is well known that in the Starobinsky inflation $\left(R^{2}\right.$ inflation) [5], $n_{\mathrm{s}}$ and $r$ are expressed as [11] Eq. (3.2) and $r=12 / N^{2}$, respectively. If $N=60$, we find $n_{\mathrm{s}}=0.967$ and $r=3.33 \times 10^{-3}$, which are consistent with the Planck data [7] (for a recent detailed review of inflation in modified gravity theories, see, for instance, [43]). The relation (3.2) can be satisfied also in the chaotic inflation [44] and the Higgs inflation with its non-minimal gravitational coupling $[45,46]$, or the so-called $\alpha$-attractor [47-49], which connects the Starobinsky, quadratic chaotic, and Higgs inflations. By combining the relation (3.2) with the first equation in (3.1), we find

$V(N)=\frac{1}{\left(C_{1} / N\right)+C_{2}}$,

with $C_{1}(>0)$ and $C_{2}$ constants, the mass dimension of which is four. For the potential $V(N)$ in Eq. (3.3), from the second relation in (3.1), the tensor-to-scalar ratio $r$ of the density perturbations is expressed as

$r=\frac{8}{N\left[1+\left(C_{2} / C_{1}\right) N\right]}$,

Furthermore, with the third relation in (3.1), the running $\alpha_{\mathrm{s}}$ of the spectral index is written as

$\alpha_{\mathrm{s}}=-\frac{2}{N^{2}}$

By using this expression, for $N=60$, we acquire $\alpha_{\mathrm{s}}=$ $-5.56 \times 10^{-4}$. This value is consistent with the Planck analysis.

In a fluid model, instead of the inflaton potential $V$, we use the EoS in Eq. (2.5). In the FLRW background, the Friedmann equation (2.6) is written as $\left(3 / \kappa^{2}\right)(H(N))^{2}=$ $\rho(N) \approx V(N)$, where the last approximate equation follows from the slow-roll approximation that the kinetic term is much smaller than the potential one as $\left|(1 / 2) \dot{\phi}^{2}\right| \ll V$. From the relation $\rho \approx V$ with Eq. (3.3), we have

$N \approx \frac{C_{1} \rho}{1-C_{2} \rho}$

Furthermore, it follows from the Friedmann equation with the slow-roll approximation shown above that the Hubble parameter is expressed as

$H(N) \approx \kappa \sqrt{\frac{1}{3\left[\left(C_{1} / N\right)+C_{2}\right]}}$, 
where $\left(C_{1} / N\right)+C_{2}>0$. From Eqs. (2.6) and (2.7) with the Hubble parameter in Eq. (3.7), we obtain

$P=-\rho-\frac{2}{\kappa^{2}} H(N) H^{\prime}(N) \approx-\rho-\frac{3 C_{1}}{N^{2} \kappa^{4}} H^{4}$.

By comparing Eq. (2.5) with Eq. (3.8), we acquire

$f(\rho) \approx-\frac{3 C_{1}}{N^{2} \kappa^{4}} H^{4} \approx-\frac{1}{3 C_{1}}\left(1-2 C_{2} \rho+C_{2}^{2} \rho^{2}\right)$.

Here, in deriving the second approximate equality, we have used the Friedmann Eqs. (2.6) and (3.6).

\subsection{Fluid models and inflation}

Next, we explicitly show the models of a fluid, in which the values of $n_{\mathrm{s}}$ and $r$ are consistent with the Planck results. Plugging Eqs. (2.11) and (2.13), we have the form of EoS for a fluid

$P=-\rho+f(\rho)=-\rho+A \rho^{\beta}+\bar{\zeta}\left(\frac{\kappa}{\sqrt{3}}\right)^{\gamma} \rho^{\gamma / 2}$.

With the results in the preceding subsection, we decide the models parameters: $A, \bar{\zeta}, \beta$, and $\gamma$, in which Eq. (3.2) can be satisfied.

\subsubsection{Case (i): $\left|C_{2} \rho\right| \gg 1$}

When $\left|C_{2} \rho\right| \gg 1$, from Eq. (3.9), we have

$f(\rho) \approx \frac{2 C_{2}}{3 C_{1}} \rho-\frac{C_{2}^{2}}{3 C_{1}} \rho^{2}$.

Since the value of $N$ given by Eq. (3.6) has to be positive, we find $C_{2}<0$. In addition, the number of $e$-folds $N$ during inflation has to be much larger than unity such as $N=60$, and hence, from Eq. (3.6) and the condition $\left|C_{2} \rho\right| \gg 1$, we acquire $\left(-C_{2}\right) / C_{1} \approx 1 / N \ll 1$. From Eqs. (3.10) and (3.11), we get

$$
\begin{aligned}
w=\frac{P}{\rho} & \approx-1-\frac{2}{3}\left(-\frac{C_{2}}{C_{1}}\right)+\frac{1}{3}\left(-\frac{C_{2}}{C_{1}}\right)\left(-C_{2} \rho\right) \\
& \approx-1+\frac{1}{3 N}\left(-2-C_{2} \rho\right)
\end{aligned}
$$

where in deriving the second approximate equality, we have used $\left(-C_{2}\right) / C_{1} \approx 1 / N$. For example, if $\left|C_{2} \rho\right|=\mathcal{O}(10)$ and $\left(-C_{2}\right) / C_{1} \approx 1 / N$, where, e.g., $N \gtrsim 60$, from Eq. (3.12), we can obtain $w \approx-1$. This implies that the slow-roll inflation, namely, the de Sitter inflation, can occur, and hence the scale factor can be represented as

$a(t)=a_{\mathrm{i}} \exp \left[H_{\mathrm{inf}}\left(t-t_{\mathrm{i}}\right)\right]$.
Moreover, if $\left(-C_{2}\right) / C_{1}<1 / N$, from Eq. (3.4), it is seen that for $N \gtrsim 73$, the tensor-to-scalar ratio $r$ of the density perturbations can meet $r<0.11$, which is consistent with the Planck results.

Through the comparison between this expression and Eq. (2.13), we see that these expressions become equivalent, i.e., the linear combination of $\rho$ and $\rho^{2}$. In this case, there are two combinations of the model parameters, which will be called Model (a) and Model (b), as follows:

Model (a): $\quad A=\frac{2 C_{2}}{3 C_{1}}, \quad \bar{\zeta}=-\frac{3 C_{2}^{2}}{C_{1} \kappa^{4}}, \quad \beta=1, \quad \gamma=4$,

and

Model (b) : $\quad A=-\frac{C_{2}^{2}}{3 C_{1}}, \quad \bar{\zeta}=\frac{2 C_{2}}{C_{1} \kappa^{2}}, \quad \beta=2, \quad \gamma=2$.

In (3.14) and (3.15), when the second relations have been derived by using the fourth relations. As a result, the EoS of a fluid can explicitly be reconstructed.

\subsubsection{Case (ii): $\left|C_{2} \rho\right| \ll 1$}

On the other hand, if $\left|C_{2} \rho\right| \ll 1$, it follows from Eq. (3.9) that

$f(\rho) \approx-\frac{1}{3 C_{1}}+\frac{2 C_{2}}{3 C_{1}} \rho$

With Eq. (3.6) and the condition $\left|C_{2} \rho\right| \ll 1$, we have $C_{1} \rho \approx N \gg 1$, and eventually we also find $\left|C_{2}\right| / C_{1} \ll 1$. From Eqs. (3.10) and (3.11), we acquire

$w=\frac{P}{\rho} \approx-1-\frac{1}{3} \frac{1}{C_{1} \rho}+\frac{2}{3}\left(\frac{C_{2}}{C_{1}}\right) \approx-1+\frac{1}{3}\left(-\frac{1}{N}+2 \frac{C_{2}}{C_{1}}\right)$.

Here, the second approximate equality follows from $C_{1} \rho \approx N$. Accordingly, from Eq. (3.17) with $1 / N \ll 1$ and $\left|C_{2}\right| / C_{1} \ll 1$, we see that $w \approx-1$ can be met. Thus, the slow-roll (de Sitter) inflation can happen, and the scale factor can be expressed by Eq. (3.13). In addition, for $C_{2}>0$ and $C_{2} / C_{1} \lesssim 1 / N$, by using Eq. (3.4), it is found that even for $N \gtrsim 60$, the tensor-to-scalar ratio $r$ of the density perturbations becomes $r<0.11$, which is consistent with the Planck results. On the other hand, for $C_{2}<0$ and $\left|C_{2}\right| / C_{1}<1 / N$, it follows from Eq. (3.4) that for $N \gtrsim 73$, we can get $r<0.11$, similarly to that in Case (i) described above.

The comparison of this expression with Eq. (2.13) leads to the following combinations of the model parameters, which 
Table 1 Fluid models with the EoS in Eq. (3.10) realizing the relation (3.2). Here, $C_{1}>0$. In Case (i), $\left|C_{2} \rho\right| \gg 1$ and $C_{2}<0$, whereas for Case (ii), $\left|C_{2} \rho\right| \ll 1$ and $C_{2}$ can take both the positive and the negative values

\begin{tabular}{llllll}
\hline Case & Model & $A$ & $\bar{\zeta}$ & $\beta$ & $\gamma$ \\
\hline (i) & (a) & $2 C_{2} /\left(3 C_{1}\right)$ & $-3 C_{2}^{2} /\left(C_{1} \kappa^{4}\right)$ & 1 & 4 \\
(i) & (b) & $-C_{2}^{2} /\left(3 C_{1}\right)$ & $2 C_{2} /\left(C_{1} \kappa^{2}\right)$ & 2 & 2 \\
(ii) & (c) & $-1 /\left(3 C_{1}\right)$ & $2 C_{2} /\left(C_{1} \kappa^{2}\right)$ & 0 & 2 \\
(ii) & (d) & $2 C_{2} /\left(3 C_{1}\right)$ & $-1 /\left(3 C_{1}\right)$ & 1 & 0 \\
\hline
\end{tabular}

will be named Model (c) and Model (d) as follows:

Model (c): $\quad A=-\frac{1}{3 C_{1}}, \quad \bar{\zeta}=\frac{2 C_{2}}{C_{1} \kappa^{2}}, \quad \beta=0, \quad \gamma=2$,

and

Model (d): $\quad A=\frac{2 C_{2}}{3 C_{1}}, \quad \bar{\zeta}=-\frac{1}{3 C_{1}}, \quad \beta=1, \quad \gamma=0$.

In Eqs. (3.18) and (3.19), with the fourth relations, the second ones have been derived. In Table 1, the fluid models with the EoS in Eq. (3.10) satisfying the relation (3.2) are summarized.

We remark that if $C_{2}>0$, the inflaton potential can correspond to the one in the Starobinsky inflation. From the investigations in the scalar field theories, we have $C_{2}=$ (2/3) $C_{1}$ [36]. In this case, for the models in Eqs. (3.18) and (3.19), we obtain $\bar{\zeta}=4 /\left(3 \kappa^{2}\right)$ and $A=4 / 9$, respectively.

\section{Graceful exit from inflation}

In this section, we examine whether the graceful exit from inflation can occur in a fluid model. We analyze the instability of the de Sitter solution $\left(H=H_{\text {inf }}(>0)=\right.$ constant $)$ during inflation by taking the perturbations of the Hubble parameter as follows [50]:

$H=H_{\mathrm{inf}}+H_{\mathrm{inf}} \delta(t)$

Here, $|\delta(t)| \ll 1$, and hence $H_{\text {inf }} \delta(t)$ denotes the perturbations from the de Sitter solution $H_{\text {inf }}$.

We rewrite Eq. (2.8) as the following second differential equation with respect to the cosmic time $t$ :

$$
\begin{aligned}
\ddot{H} & -\frac{\kappa^{4}}{2}\left[\beta A^{2}\left(\frac{3}{\kappa^{2}}\right)^{2 \beta} H^{4 \beta-1}+\left(\beta+\frac{\gamma}{2}\right) A \bar{\zeta}\left(\frac{3}{\kappa^{2}}\right)^{\beta}\right. \\
& \left.\times H^{2 \beta+\gamma-1}+\frac{\gamma}{2} \bar{\zeta}^{2} H^{2 \gamma-1}\right]=0 .
\end{aligned}
$$

We define the form of $\delta(t)$ as

$\delta(t) \equiv \mathrm{e}^{\lambda t}$,

where $\lambda$ is a constant, so that we can investigate the instability of the de Sitter solution. If there is a positive solution of $\lambda$, the de Sitter solution can be unstable. Therefore, the universe can exit from inflation, and the reheating stage can follow, because the absolute value of $\delta(t)$ with $\lambda>0$ becomes larger as the cosmic time grows at the inflationary stage.

We substitute Eq. (4.1) with Eq. (4.3) into Eq. (4.2) and take the first order of $\delta(t)$. Accordingly, we get

$$
\begin{aligned}
\lambda^{2} & -\frac{1}{2} \frac{\kappa^{4}}{H_{\mathrm{inf}}^{2}} \mathcal{Q}=0, \\
\mathcal{Q} & \equiv \beta(4 \beta-1) A^{2}\left(\frac{3}{\kappa^{2}}\right)^{2 \beta} H_{\mathrm{inf}}^{4 \beta} \\
& +\left(\beta+\frac{\gamma}{2}\right)(2 \beta+\gamma-1) A \bar{\zeta}\left(\frac{3}{\kappa^{2}}\right)^{\beta} H_{\mathrm{inf}}^{2 \beta+\gamma} \\
& +\frac{\gamma}{2}(2 \gamma-1) \bar{\zeta}^{2} H_{\mathrm{inf}}^{2 \gamma} .
\end{aligned}
$$

We see that the solutions of Eq. (4.4) are given by

$\lambda=\lambda_{ \pm} \equiv \pm \frac{1}{\sqrt{2}} \frac{\kappa^{2}}{H_{\text {inf }}} \sqrt{\mathcal{Q}}$.

If $\mathcal{Q}>0$, we can acquire the positive solution of $\lambda=$ $\lambda_{+}>0$. As a result, the exit from inflation can gracefully occur.

Concretely, in the fluid models reconstructed above and summarized in Table 1, we check whether the graceful exit from inflation can be realized or not, namely, whether $\mathcal{Q}$ can take a positive value or not. If the universe cannot successfully exit from inflation, inflation does not ends, and therefore such a scenario corresponds to the so-called eternal inflation. By substituting the values of $A, \bar{\zeta}, \beta$, and $\gamma$ in Models (a), (b), (c), and (d), given by Eqs. (3.14), (3.15), (3.18), and (3.19), respectively, into Eq. (4.6), we obtain the expressions of $\mathcal{Q}$ in each models. To evaluate the values of $\mathcal{Q}$, we take into account the following facts. For all of the models, $C_{1}>0$. On the other hand, in Models (a) and (b), $C_{2}<0$, while in Models (c) and (d), $C_{2}$ can become both the positive and the negative values. For Models (a) and (b) in Case (i), we find

$$
\begin{aligned}
& \text { Model (a): } \mathcal{Q}=2\left(\frac{C_{2}}{C_{1}}\right)^{2}\left(\frac{H_{\text {inf }}}{\kappa}\right)^{4} \\
& \times\left[6-45 C_{2}\left(\frac{H_{\text {inf }}}{\kappa}\right)^{2}+63 C_{2}^{2}\left(\frac{H_{\text {inf }}}{\kappa}\right)^{4}\right]>0,
\end{aligned}
$$


Table 2 The EoS for the fluid models reconstructed in Sect. III and the conditions that in these models, the graceful exit from inflation can be realized. In these models, $n_{\mathrm{s}}-1=-2 / N=0.967$ for $N=60$, $r<0.11$ for $N \gtrsim 73$ in Models (a), (b) and Models (c) and (d) with $C_{2}<0$, or $N \gtrsim 60$ in Models (c) and (d) with $C_{2}>0$, and $\alpha_{\mathrm{s}}=-2 / N^{2}=-5.56 \times 10^{-4}$ for $N=60$ can be realized. These values can explain the Planck data. Legend is the same as Table 1

\begin{tabular}{llll}
\hline Case & Model & EoS & Conditions for the graceful exit from inflation \\
\hline (i) & (a) & $P=-\rho+\left[2 C_{2} /\left(3 C_{1}\right)\right] \rho-\left[3 C_{2}^{2} /\left(C_{1} \kappa^{4}\right)\right] H^{4}$ & No condition \\
(i) & (b) & $P=-\rho-\left[C_{2}^{2} /\left(3 C_{1}\right)\right] \rho^{2}+\left[2 C_{2} /\left(C_{1} \kappa^{2}\right)\right] H^{2}$ & No condition \\
(ii) & (c) & $P=-\rho-\left[1 /\left(3 C_{1}\right)\right]+\left[2 C_{2} /\left(C_{1} \kappa^{2}\right)\right] H^{2}$ & $C_{2}<0$ or $C_{2}>(1 / 36)\left(\kappa / H_{\text {inf }}\right)^{2}$ \\
(ii) & (d) & $P=-\rho+\left[2 C_{2} /\left(3 C_{1}\right)\right] \rho-\left[1 /\left(3 C_{1}\right)\right]$ & $C_{2}<0$ or $C_{2}>(1 / 18)\left(\kappa / H_{\text {inf }}\right)^{2}$ \\
\hline
\end{tabular}

Model (b) $: \mathcal{Q}=6\left(\frac{C_{2}}{C_{1}}\right)^{2}\left(\frac{H_{\text {inf }}}{\kappa}\right)^{4}$

$$
\times\left[2-15 C_{2}\left(\frac{H_{\mathrm{inf}}}{\kappa}\right)^{2}+21 C_{2}^{2}\left(\frac{H_{\mathrm{inf}}}{\kappa}\right)^{4}\right]>0 .
$$

Accordingly, we always have $\mathcal{Q}>0$. While, for Models (c) and (d) in Case (ii), we acquire

$$
\begin{gathered}
\text { Model (c): } \mathcal{Q}=\left(\frac{C_{2}}{C_{1}}\right)^{2}\left(\frac{H_{\text {inf }}}{\kappa}\right)^{2} \\
\times\left[-\frac{1}{3 C_{2}}+12\left(\frac{H_{\text {inf }}}{\kappa}\right)^{2}\right],
\end{gathered}
$$

Model $(\mathrm{d}): \quad \mathcal{Q}=2\left(\frac{C_{2}}{C_{1}}\right)^{2}\left(\frac{H_{\text {inf }}}{\kappa}\right)^{2}$

$$
\times\left[6\left(\frac{H_{\mathrm{inf}}}{\kappa}\right)^{2}-\frac{1}{3 C_{2}}\right] \text {. }
$$

From these relations, we find that if $C_{2}<0$, we get $\mathcal{Q}>0$, whereas, in the case that $C_{2}>0$, if the following conditions are satisfied:

$C_{2}>\frac{1}{36}\left(\frac{\kappa}{H_{\text {inf }}}\right)^{2}$ for Model (c),

$C_{2}>\frac{1}{18}\left(\frac{\kappa}{H_{\text {inf }}}\right)^{2}$ for Model (d),

we can obtain $\mathcal{Q}>0$. Thus, for the reconstructed models of a fluid in the previous section, it is possible for the universe to gracefully exit from inflation.

In Table 2, we present the summary of the reconstructed fluid models. We show the EoS of these models in the form of Eq. (2.11) so that a term inspired by bulk viscosity can clearly be seen. In these models, the three observables of inflationary cosmology can be compatible with the Planck results. First, the spectral index $n_{\mathrm{s}}$ of the curvature perturbations is expressed as $n_{\mathrm{s}}-1=-2 / N$ in Eq. (3.2), which can lead to 0.967 for $N=60$. Second, The tensor-to-scalar ratio $r$ of the density perturbations can satisfy the upper limit of $r<0.11$. In Models (a) and (b) [Case (i)] and Models (c) and (d) [Case (ii)] with $C_{2}<0$, if $N \gtrsim 73$, we can obtain $r<0.11$. While, in Models (c) and (d) [Case (ii)] with $C_{2}>0$, when $N \gtrsim 60$, we can find $r<0.11$. Third, the running $\alpha_{\mathrm{s}}$ of the spectral index is given by $\alpha_{\mathrm{s}}=-2 / N^{2}$ in Eq. (3.5). From this expression, we have $\alpha_{\mathrm{s}}=-5.56 \times 10^{-4}$. These values of $n_{\mathrm{s}}$, $r$, and $\alpha_{\mathrm{s}}$ are consistent with the Planck results. Moreover, the universe can gracefully exit from inflation. We describe the conditions for the graceful exit from inflation.

\section{Singular inflation in a fluid model}

In this section, we study the singular inflation [39] in a fluid model. In this inflationary scenario, the idea of finite-time future singularities in the context of the dark energy problem is applied to inflation in the early universe.

The finite-time future singularities are classified into four types [51]. Their features in modified gravity theories have also been analyzed in detail [52] (for a detailed review on the finite-time future singularities, see [17]). Among them, the formulation of the Type IV singularity can be used in the singular inflation, because there is no divergence in terms of the scale factor, the energy density and pressure of the universe.

In the type IV singularity, for $t \rightarrow t_{\mathrm{s}}$, where $t_{\mathrm{S}}$ is the time when the singularity appears, the scale factor $a$, the effective (i.e., total) energy density $\rho_{\text {eff }}$ and pressure $P_{\text {eff }}$ of the universe become finite as $a \rightarrow a_{\mathrm{s}}, \rho_{\mathrm{eff}} \rightarrow 0$ and $\left|P_{\text {eff }}\right| \rightarrow 0$. Here, $a_{\mathrm{s}}$ is the value of $a$ at $t=t_{\mathrm{s}}$. The case that $\rho_{\text {eff }}$ and/or $\left|P_{\text {eff }}\right|$ become non-zero finite values at $t=t_{\mathrm{S}}[53-$ $80]$ is also included in the Type IV singularity. However, the higher derivatives of $H$ diverge.

In the following, we explore the inflationary stage in which there is only a component of a fluid. Therefore, for simplicity, we describe $\rho_{\text {eff }}$ and $P_{\text {eff }}$ by $\rho$ and $P$, respectively. We consider the case that the Hubble parameter and scale factor during inflation are represented as

$$
\begin{aligned}
H & =H_{\mathrm{inf}}+\bar{H}\left(t_{\mathrm{s}}-t\right)^{q}, \quad q>1, \\
a & =\bar{a} \exp \left[H_{\mathrm{inf}} t-\frac{\bar{H}}{q+1}\left(t_{\mathrm{s}}-t\right)^{q+1}\right],
\end{aligned}
$$

where $\bar{H}, q$, and $\bar{a}$ are constants, and the mass dimension of $\bar{H}$ is $q+1$. 
In the flat FLRW universe, from the gravitational field equations, the energy density and pressure of the universe are given by

$\rho=\frac{3 H^{2}}{\kappa^{2}}, \quad P=-\frac{2 \dot{H}+3 H^{2}}{\kappa^{2}}$.

It is seen from Eq. (5.2) and the expressions in (5.3) with Eq. (5.1) that in the limit $t \rightarrow t_{\mathrm{s}}$, all of $a, \rho$, and $P$ asymptotically approach finite values, while the higher derivatives of $H$ diverge. Thus, the Type IV singularity appears at $t=t_{\mathrm{s}}$. By using the expressions of $\rho$ and $P$ in (5.2) with Eq. (5.1), we find the following EoS for a fluid:

$P=-\rho+f(\rho), \quad f(\rho)=\frac{2 q \bar{H}^{1 / q}}{\kappa^{2}}\left(\kappa \sqrt{\frac{\rho}{3}}-H_{\mathrm{inf}}\right)^{(q-1) / q}$.

Here, $f(\rho)$ can be described as the series of power of $\rho$. In fact, if $H_{\text {inf }} / \sqrt{\kappa^{2} \rho / 3}=H_{\text {inf }} / H \ll 1$, where the equality comes from the first equation in (5.3), we find

$$
\begin{aligned}
f(\rho) \approx & \frac{2}{3^{(q-1) /(2 q)}} \frac{\bar{H}^{1 / q}}{\kappa^{(q+1) / q}} \\
& \times\left[\rho^{(q-1) /(2 q)}-\frac{\sqrt{3}(q-1)}{q} \frac{H_{\mathrm{inf}}}{\kappa} \rho^{-1 /(2 q)}\right],
\end{aligned}
$$

where we have taken the first order of the quantity $\left(H_{\text {inf }} / \sqrt{\left(\kappa^{2} \rho\right) / 3}\right)$. It follows from Eq. (5.5) that $f(\rho)$ is represented as a linear combination of two powers of $\rho$, similarly to that in Eq. (2.13) or (3.11). Hence, this model can be regarded as a kind of the fluid models reconstructed in Sect. 3.

Furthermore, Eq. (5.5) divided by $\rho$ reads

$$
\begin{aligned}
\frac{f(\rho)}{\rho} \approx & \frac{2 q}{3} \bar{H}^{1 / q}\left(\frac{\kappa^{2} \rho}{3}\right)^{-(q+1) /(2 q)} \\
& \times\left[1-\frac{(q-1)}{q} \frac{H_{\mathrm{inf}}}{\sqrt{\kappa^{2} \rho / 3}}\right] \\
= & \frac{2 q}{3}\left(\frac{\bar{H}}{H^{q+1}}\right)^{1 / q}\left[1-\frac{(q-1)}{q} \frac{H_{\mathrm{inf}}}{H}\right] .
\end{aligned}
$$

Here, in deriving the last equality, we have used the first equation in (5.3). It is seen from Eq. (5.6) that for $\bar{H} / H^{q+1} \ll$ 1 , we have $f(\rho) / \rho \ll 1$. In this case, the observables of the inflationary models, i.e., $n_{\mathrm{s}}, r$, and $\alpha_{\mathrm{s}}$, can approximately be represented by Eq. (2.9), and the values of $n_{\mathrm{s}}, r$, and $\alpha_{\mathrm{s}}$ can be compatible with the Planck analysis, as stated in Sect. 2.3.

We explain the existence of limit of $\bar{\zeta}=0$ in Eq. (2.12), in which the term $\zeta(H)$ in Eq. (2.12) will not exist and therefore the EoS for a fluid in Eq. (2.11) reads $P=-\rho+A \rho^{\beta}$. In such a limit, from Eq. (2.5), we have $f(\rho)=A \rho^{\beta}$, namely, the term $f(\rho)$ consists of the single power of $\rho$. On the other hand, in Eqs. (5.5) and (5.6), the form of $f(\rho)$ is a linear combination of two kinds of power of $\rho$. The form of $f(\rho)$ can approximately be given in Eqs. (5.5) and (5.6) only if the singular inflation occurs and the Hubble parameter and the scale factor are expressed as Eqs. (5.1) and (5.2), respectively, This means that for a fluid without the term $\zeta(H)$ in Eq. (2.12), the singular inflation cannot be realized. Thus, the existence of the term $\zeta(H)$ can influence the dynamics of the universe filled with a fluid in the early universe.

As a consequence, it is considered that the singular inflation can be realized in the fluid models in which the spectral index of the curvature perturbations can explain the recent Planck results.

\section{Equivalence between a fluid description of inflation and the description of inflation in terms of scalar field theories}

In this section, we demonstrate that a fluid description of inflation can be equivalent to the description of inflation in terms of scalar field theories (for further related investigations, see Ref. [17]). The action of scalar field theories is expressed as

$S=\int d^{4} x \sqrt{-g}\left(\frac{R}{2 \kappa^{2}}-\frac{1}{2} \omega(\varphi) \partial_{\mu} \varphi \partial^{\mu} \varphi-V(\varphi)\right)$.

Here, $\omega(\varphi)$ is a coefficient function of kinetic term of the scalar field $\varphi$ and $V(\varphi)$ is the potential of $\varphi$. Starting from a fluid description, we construct a scalar field theory with the same EoS as that of a fluid. By this process, we obtain the expressions of $\omega(\varphi)$ and $V(\varphi)$ of the corresponding scalar field theory to a fluid description. Consequently, we can represent a fluid description as the description of a scalar field theory.

It is well known that in the FLRW background, $\omega(\varphi)$ and $V(\varphi)$ can be described as [81]

$\begin{aligned} \omega(\varphi) & =-\frac{2}{\kappa^{2}} \frac{\mathrm{d} J(\varphi)}{\mathrm{d} \varphi}, \\ V(\varphi) & =\frac{1}{\kappa^{2}}\left[3(J(\varphi))^{2}+\frac{\mathrm{d} J(\varphi)}{\mathrm{d} \varphi}\right],\end{aligned}$

with $J(\varphi)$ an arbitrary function of $\varphi$. Here, we can take $\varphi=t$ and $H=J(t)$ because $\varphi$ can be treated as an auxiliary scalar quantity. On the other hand, the energy density $\rho$ and pressure $P$ of the scalar field $\varphi$ read

$$
\begin{aligned}
\rho & =\frac{1}{2} \omega(\varphi) \dot{\varphi}^{2}+V(\varphi), \\
P & =\frac{1}{2} \omega(\varphi) \dot{\varphi}^{2}-V(\varphi) .
\end{aligned}
$$


With these equations, we find that $\omega(\varphi)$ and $V(\varphi)$ are given by

$$
\begin{aligned}
\omega \dot{\varphi}^{2} & =\rho+P=f(\rho), \\
V & =\frac{1}{2}(\rho-P)=\rho-\frac{f(\rho)}{2} .
\end{aligned}
$$

In deriving the second equalities in Eqs. (6.6) and (6.7), we have used the EoS of $P=-\rho+f(\rho)$ in a fluid description in Eq. (2.5). From the Friedmann Eq. (2.6), we find $\rho=3 H^{2} / \kappa^{2}$. Therefore, when we have $H(=I(t))$, we can express $\rho=\rho(t(\varphi))=\rho(\varphi)$ as a function of $t(=\varphi)$. Eventually, from Eqs. (6.6) and (6.7) with $\rho=\rho(\varphi)$, we can acquire the expressions of $\omega=\omega(\varphi)$ and $V=V(\varphi)$. By using these processes, we can obtain the description of a scalar field theory corresponding to an original fluid description.

Moreover, we consider the opposite approach from the description of a scalar field to a fluid description. We first have a scalar field action with $\omega(\varphi)$ and $V(\varphi)$ in Eq. (6.1). It follows from Eqs. (6.4) and (6.5) with $\phi=t$ and $H=J(t)$ that the EoS of the universe $w \equiv P / \rho=-1+f(\rho) / \rho$ in Eq. (6.1) with Eq. (2.5). Plugging this relation with Eq. (6.4) and comparing the relation obtained with $w=-1+f(\rho) / \rho$, we get $f(\rho)$ in a fluid description. Thus, both approaches shown above suggest the equivalence between a fluid description and the description in terms of scalar field theories.

As a fluid description, we address the case that $f(\rho)$ is given by

$f(\rho)=\bar{f}_{1}+\bar{f}_{2}\left(\frac{\rho}{\rho_{*}}\right)^{u}$,

where $\bar{f}_{1}, \bar{f}_{2}$, and $u$ are constants, and $\rho_{*}$ is a fiducial value of $\rho$ For $f(\rho)$ in Eq. (6.8) with $u=1$, we have the following EoS:

$P=-\rho+\left(\frac{\bar{f}_{2}}{\rho_{*}}\right) \rho+\bar{f}_{1}$,

By comparing this form with Model (d) in Table 2, it is seen that if $\bar{f}_{2} / \rho_{*}=2 C_{2} /\left(3 C_{1}\right)$ and $\bar{f}_{1}=-\left[1 /\left(3 C_{1}\right)\right]$, the form of the EoS in Eq. (6.9) is equal to Model (d). This means that in a fluid description with the form of the EoS in Eq. (6.8), the spectral index of the curvature perturbations, the tensor-to-scalar ratio of the density perturbations, and the running of the spectral index can be consistent with the Planck results.

From the substitution of Eq. (6.8) into the relation $w=$ $-1+f(\rho) / \rho$, Eqs. (6.6) and (6.7), we obtain

$$
w=-1+\frac{\bar{f}_{1}}{\rho}+\frac{\bar{f}_{2}}{\rho_{*}}\left(\frac{\rho}{\rho_{*}}\right)^{u-1},
$$

$$
\begin{aligned}
\omega \dot{\varphi}^{2} & =\bar{f}_{1}+\bar{f}_{2}\left(\frac{\rho}{\rho_{*}}\right)^{u}, \\
V & =\rho-\frac{1}{2}\left[\bar{f}_{1}+\bar{f}_{2}\left(\frac{\rho}{\rho_{*}}\right)^{u}\right] .
\end{aligned}
$$

In addition, for instance, we consider the case that the Hubble parameter and the scale factor during inflation are expressed by

$$
\begin{aligned}
H & =\frac{\bar{h}}{t}, \\
a & =\tilde{a} t^{\bar{h}},
\end{aligned}
$$

where $\bar{h} \gg 1$ and $\tilde{a}(\neq 0)$ are constants. Such a case of $\bar{h} \gg 1$ corresponds to the quasi-de Sitter inflation (i.e., the slow-roll inflation). In this case, it follows from Eqs. (6.2) and (6.3) with $\varphi=t$ and $H=J(t)$ that

$$
\begin{aligned}
\omega(\varphi) & =\frac{2 \bar{h}}{\kappa^{2}} \frac{1}{\varphi^{2}}, \\
V(\varphi) & =\frac{\bar{h}(3 \bar{h}-1)}{\kappa^{2}} \frac{1}{\varphi^{2}} .
\end{aligned}
$$

As described above, when the Hubble parameter $H$ can be represented as a function of $t$, from Eqs. (6.2) and (6.3) with $\varphi=t$ and $H=J(t)$, the expressions of $\omega=\omega(\varphi)$ and $V=V(\varphi)$ can be derived explicitly.

\section{Conclusions}

In the present paper, we have investigated the description of the inflationary universe in the framework of a fluid model in which the EoS for a fluid includes bulk viscosity. It has been found that in a fluid description, the three observables of inflationary models, namely, the spectral index $n_{\mathrm{s}}$ of the curvature perturbations, the tensor-to-scalar ratio $r$ of the density perturbations, and the running $\alpha_{\mathrm{s}}$ of the spectral index, can be consistent with the recent Planck results.

Furthermore, we have explicitly reconstructed the EoS of a fluid model from the spectral index $n_{\mathrm{s}}$ of the curvature perturbations. Particularly, we have used the expression of $n_{\mathrm{s}}$ as a function of the number of $e$-folds $N$ in the inflationary models, where the value of $n_{\mathrm{s}}$ can explain the Planck data, including the Starobinsky inflation. It has been shown that for the fluid models reconstructed from the spectral index, indeed, the slow-roll (de Sitter) inflation can occur. It has also been certified that, in these fluid models, the tensor-toscalar ratio $r$ of the density perturbations can meet the upper limit found by the Planck analysis. The running $\alpha_{\mathrm{s}}$ of the spectral index can be compatible with the Planck results.

In our previous work [16], since we have considered a fluid without the term $\zeta(H)$ in Eq. (2.12), only for the special case that the EoS for a fluid is approximately equal to -1 as $w=P / \rho \approx-1$, it has been shown that in a fluid model, the 
three observables of inflationary models can be consistent with the Planck results. On the other hand, in this work, we have introduced the additional term $\zeta(H)$ in Eq. (2.12) into the EoS for a fluid as in Eq. (2.11). As a result, it has been found that also for cases in which the value of $w$ for the EoS of a fluid is different from -1 , in such a fluid model, the three observables of inflationary models can be compatible with the Planck analysis.

In addition, we have examined the instability of the de Sitter solution at the inflationary stage by analyzing the perturbations of the Hubble parameter. It has been performed that the universe can gracefully exit from inflation in the reconstructed models of a fluid. We have also derived the conditions for the graceful exit from inflation to be realized in the reconstructed fluid models.

Moreover, we have explored the singular inflation in a fluid model by using the formulations to describe the type IV singularity, which is one of the four types of the finite-time future singularity. It has been demonstrated that the singular inflation can be realized in the fluid models where the spectral index of the curvature perturbations can be compatible with the Planck data.

It has also been studied that a fluid description of inflation can be equivalent to the description of inflation in terms of scalar field theories.

Consequently, not only the representation of inflation in scalar field theories but also a fluid description of the inflationary universe can explain the observational results acquired by the Planck satellite.

The present method of the reconstruction may equally be applied in the case that the universe is filled with several coupled fluids. This description may also be applied to the cosmological evolution from modified gravity consistent with a fluid description at the background evolution level.

Acknowledgments This work was partially supported by the JSPS Grant-in-Aid for Young Scientists (B) \# 25800136 and the researchfunds provided by Fukushima University (K.B.), and by MINECO (Spain) project FIS2013-44881 and I-LINK 1019 and JSPS fellowship ID No.:S15127 (S.D.O.).

Open Access This article is distributed under the terms of the Creative Commons Attribution 4.0 International License (http://creativecomm ons.org/licenses/by/4.0/), which permits unrestricted use, distribution, and reproduction in any medium, provided you give appropriate credit to the original author(s) and the source, provide a link to the Creative Commons license, and indicate if changes were made.

Funded by SCOAP ${ }^{3}$.

\section{Appendix A: Slow-roll parameters in a fluid description}

In this appendix, we present the slow-roll parameters in a fluid description. The explicit expressions are given by

$$
\epsilon=\frac{3}{2} \rho(N) f(\rho)\left(\frac{f^{\prime}(\rho)-2}{2 \rho(N)-f(\rho)}\right)^{2},
$$

$$
\begin{aligned}
\eta= & \frac{3 \rho(N)}{2 \rho(N)-f(\rho)}\left\{\frac{f(\rho)}{\rho(N)}+\frac{1}{2}\left(f^{\prime}(\rho)\right)^{2}+f^{\prime}(\rho)\right. \\
& -\frac{5}{2} \frac{f(\rho) f^{\prime}(\rho)}{\rho(N)}+\left(\frac{f(\rho)}{\rho(N)}\right)^{2} \\
& +\frac{1}{3} \frac{\rho^{\prime}(N)}{f(\rho)}\left[\left(f^{\prime}(\rho)\right)^{2}+f(\rho) f^{\prime \prime}(\rho)\right. \\
& \left.\left.-2 \frac{f(\rho) f^{\prime}(\rho)}{\rho(N)}+\left(\frac{f(\rho)}{\rho(N)}\right)^{2}\right]\right\}, \\
\xi^{2}= & \frac{f(\rho) \rho(N)\left(f^{\prime}(\rho)-2\right)}{2(2 \rho(N)-f(\rho))^{2}}\left[\frac{45}{2} \frac{f(\rho)}{\rho(N)}\left(f^{\prime}(\rho)-\frac{1}{2} \frac{f(\rho)}{\rho(N)}\right)\right. \\
& +18\left(\frac{f(\rho)}{\rho(N)}\right)^{-1}\left(f^{\prime}(\rho)-\frac{1}{2} \frac{f(\rho)}{\rho(N)}\right)^{2} \\
& +18\left(\frac{f(\rho)}{\rho(N)}\right)^{-1}\left(f^{\prime}(\rho)-\frac{1}{2} \frac{f(\rho)}{\rho(N)}\right)^{3} \\
& \left.-9\left(f^{\prime}(\rho)-\frac{1}{2} \frac{f(\rho)}{\rho(N)}\right)^{2}-45 f^{\prime}(\rho)+9 \frac{f(\rho)}{\rho(N)}+I\right],
\end{aligned}
$$

where

$$
\begin{aligned}
& I \equiv 3\left(4 f^{\prime}(\rho)-7 \frac{f(\rho)}{\rho(N)}+2\right)\left\{-\frac{3}{2}\left(f^{\prime}(\rho)-\frac{1}{2} \frac{f(\rho)}{\rho(N)}\right)\right. \\
& +\left(\frac{f(\rho)}{\rho(N)}\right)^{-2} \frac{\rho^{\prime}(N)}{\rho(N)}\left[\left(f^{\prime}(\rho)\right)^{2}+f(\rho) f^{\prime \prime}(\rho)-2 \frac{f(\rho) f^{\prime}(\rho)}{\rho(N)}\right. \\
& \left.\left.+\left(\frac{f(\rho)}{\rho(N)}\right)^{2}\right]\right\} \\
& +2\left(\frac{f(\rho)}{\rho(N)}\right)^{-2}\left\{-\frac{3}{2}\left(\frac{f(\rho)}{\rho(N)}\right)\left(\frac{\rho^{\prime}(N)}{\rho(N)}\right)\right. \\
& \times\left[3\left(f^{\prime}(\rho)\right)^{2}+2 f(\rho) f^{\prime \prime}(\rho)-\frac{11}{2} \frac{f(\rho) f^{\prime}(\rho)}{\rho(N)}+\frac{5}{2}\left(\frac{f(\rho)}{\rho(N)}\right)^{2}\right] \\
& +\left(\frac{\rho^{\prime \prime}(N)}{\rho(N)}\right)\left[\left(f^{\prime}(\rho)\right)^{2}+f(\rho) f^{\prime \prime}(\rho)-2 \frac{f(\rho) f^{\prime}(\rho)}{\rho(N)}+\left(\frac{f(\rho)}{\rho(N)}\right)^{2}\right] \\
& +\left(\frac{\rho^{\prime}(N)}{\rho(N)}\right)^{2}\left[\left(3 f^{\prime}(\rho) f^{\prime \prime}(\rho)+f(\rho) f^{\prime \prime \prime}(\rho)\right) \rho(N)-3\left(f^{\prime}(\rho)\right)^{2}\right. \\
& \left.\left.-3 f(\rho) f^{\prime \prime}(\rho)+6 \frac{f(\rho) f^{\prime}(\rho)}{\rho(N)}-3\left(\frac{f(\rho)}{\rho(N)}\right)^{2}\right]\right\} .
\end{aligned}
$$

\section{References}

1. K. Sato, Mon. Not. Roy. Astron. Soc. 195, 467 (1981)

2. A.H. Guth, Phys. Rev. D 23, 347 (1981)

3. A.D. Linde, Phys. Lett. B 108, 389 (1982)

4. A. Albrecht, P.J. Steinhardt, Phys. Rev. Lett. 48, 1220 (1982)

5. A.A. Starobinsky, Phys. Lett. B 91, 99 (1980)

6. P.A.R. Ade et al. [Planck Collaboration]. arXiv:1502.01589 [astroph.CO]

7. P.A.R. Ade et al. [Planck Collaboration]. arXiv:1502.02114 [astroph.CO]

8. P.A.R. Ade et al. [BICEP2 Collaboration], Phys. Rev. Lett. 112, 241101 (2014). arXiv:1403.3985 [astro-ph.CO] 
9. P.A.R. Ade et al. [BICEP2 and Planck Collaborations], Phys. Rev. Lett. 114, 101301 (2015). arXiv:1502.00612 [astro-ph.CO]

10. E. Komatsu et al. [WMAP Collaboration], Astrophys. J. Suppl. 192, 18 (2011). arXiv:1001.4538 [astro-ph.CO]

11. G. Hinshaw et al. [WMAP Collaboration], Astrophys. J. Suppl. 208, 19 (2013). arXiv:1212.5226 [astro-ph.CO]

12. J.E. Lidsey, A.R. Liddle, E.W. Kolb, E.J. Copeland, T. Barreiro, M. Abney, Rev. Mod. Phys. 69, 373 (1997). arXiv:astro-ph/9508078

13. D.H. Lyth, A. Riotto, Phys. Rept. 314, 1 (1999). arXiv:hep-ph/9807278

14. D.S. Gorbunov, V.A. Rubakov, Introduction to the theory of the early universe: cosmological perturbations and inflationary theory (World Scientific, Hackensack, 2011)

15. K. Bamba, S. Nojiri, S.D. Odintsov, Phys. Lett. B 737, 374 (2014). arXiv:1406.2417 [hep-th]

16. K. Bamba, S. Nojiri, S.D. Odintsov, D. Sáez-Gómez, Phys. Rev. D 90, 124061 (2014). arXiv:1410.3993 [hep-th]

17. K. Bamba, S. Capozziello, S. Nojiri, S.D. Odintsov, Astrophys. Space Sci. 342, 155 (2012). arXiv:1205.3421 [gr-qc]

18. S. Nojiri, S.D. Odintsov, Phys. Rept. 505, 59 (2011). arXiv:1011.0544 [gr-qc]

19. S. Nojiri, S.D. Odintsov, eConf C 0602061 (2006) 06 [Int. J. Geom. Meth. Mod. Phys. 4, 115 (2007)]. arXiv:hep-th/0601213

20. A. Joyce, B. Jain, J. Khoury, M. Trodden, Phys. Rept. 568, 1 (2015). arXiv:1407.0059 [astro-ph.CO]

21. S. Capozziello, V. Faraoni, Beyond Einstein Gravity (Springer, Dordrecht, 2010)

22. S. Capozziello, M. De Laurentis, Phys. Rept. 509, 167 (2011). arXiv:1108.6266 [gr-qc]

23. K. Koyama. arXiv:1504.04623 [astro-ph.CO]

24. A. de la Cruz-Dombriz, D. Sáez-Gómez, Entropy 14, 1717 (2012). arXiv:1207.2663 [gr-qc]

25. K. Bamba, S. Nojiri, S.D. Odintsov. arXiv:1302.4831 [gr-qc]

26. K. Bamba, S.D. Odintsov. arXiv:1402.7114 [hep-th]

27. J.D. Barrow, J.P. Mimoso, Phys. Rev. D 50, 3746 (1994)

28. I. Brevik, E. Elizalde, S. Nojiri, S.D. Odintsov, Phys. Rev. D 84, 103508 (2011). arXiv:1107.4642 [hep-th]

29. J. Wang, X. Meng, Mod. Phys. Lett. A 29, 1450009 (2014). arXiv:1303.4964 [astro-ph.CO]

30. E. Elizalde, V.V. Obukhov, A.V. Timoshkin, Mod. Phys. Lett. A 29, 1450132 (2014). arXiv: 1406.7653 [gr-qc]

31. I. Brevik, V.V. Obukhov, A.V. Timoshkin, Astrophys. Space Sci. 355, 399 (2015). arXiv:1410.2750 [gr-qc]

32. R. Myrzakulov, L. Sebastiani, Astrophys. Space Sci. 356, 205 (2015). arXiv:1410.3573 [gr-qc]

33. S. Myrzakul, R. Myrzakulov, L. Sebastiani, Astrophys. Space Sci. 357, 168 (2015). arXiv: 1504.03173 [gr-qc]

34. S. Nojiri, S.D. Odintsov, Phys. Rev. D 72, 023003 (2005). arXiv:hep-th/0505215

35. I. Brevik, Ø. Grøn, arXiv:1409.8561 [gr-qc]

36. T. Chiba. arXiv:1504.07692 [astro-ph.CO]

37. J.D. Barrow, A.A.H. Graham, Phys. Rev. D 91, 083513 (2015). arXiv: 1501.04090 [gr-qc]

38. S. Nojiri, S.D. Odintsov, V.K. Oikonomou, E.N. Saridakis, arXiv: 1503.08443 [gr-qc]

39. S. Nojiri, S.D. Odintsov, V.K. Oikonomou, Phys. Lett. B 747, 310 (2015). arXiv:1506.03307 [gr-qc]

40. S.D. Odintsov, V.K. Oikonomou. arXiv:1507.05273 [gr-qc]

41. B.A. Bassett, S. Tsujikawa, D. Wands, Rev. Mod. Phys. 78, 537 (2006). arXiv:astro-ph/0507632

42. E.W. Kolb, M.S. Turner, The early universe (Addison-Wesley, Redwood City, 1990)

43. K. Bamba, S.D. Odintsov, Symmetry 7, 220 (2015). arXiv:1503.00442 [hep-th]

44. A.D. Linde, Phys. Lett. B 129, 177 (1983)
45. D.S. Salopek, J.R. Bond, J.M. Bardeen, Phys. Rev. D 40, 1753 (1989)

46. F.L. Bezrukov, M. Shaposhnikov, Phys. Lett. B 659, 703 (2008). arXiv:0710.3755 [hep-th]

47. R. Kallosh, A. Linde, JCAP 1307, 002 (2013). arXiv:1306.5220 [hep-th]

48. R. Kallosh, A. Linde, Phys. Rev. D 91, 083528 (2015). arXiv:1502.07733 [astro-ph.CO]

49. R. Kallosh, A. Linde. arXiv:1503.06785 [hep-th]

50. K. Bamba, S.D. Odintsov, P.V. Tretyakov, Eur. Phys. J. C 75, 344 (2015). arXiv:1505.00854 [hep-th]

51. S. Nojiri, S.D. Odintsov, S. Tsujikawa, Phys. Rev. D 71, 063004 (2005). arXiv:hep-th/0501025

52. K. Bamba, S. Nojiri, S.D. Odintsov, JCAP 0810, 045 (2008). arXiv:0807.2575 [hep-th]

53. V. Sahni, Y. Shtanov, JCAP 0311, 014 (2003). arXiv:astro-ph/0202346

54. Y. Shtanov, V. Sahni, Class. Quant. Grav. 19, L101 (2002). arXiv:gr-qc/0204040

55. J.D. Barrow, Class. Quant. Grav. 21, L79 (2004) arXiv:gr-qc/0403084

56. S. Nojiri, S.D. Odintsov, Phys. Lett. B 595, 1 (2004). arXiv:hep-th/0405078

57. K. Lake, Class. Quant. Grav. 21, L129 (2004). arXiv:gr-qc/0407107

58. S. Nojiri, S.D. Odintsov, Phys. Rev. D 70, 103522 (2004). arXiv:hep-th/0408170

59. S. Cotsakis, I. Klaoudatou, J. Geom. Phys. 55, 306 (2005). arXiv:gr-qc/0409022

60. J.D. Barrow, C.G. Tsagas, Class. Quant. Grav. 22, 1563 (2005). arXiv:gr-qc/0411045

61. M.P. Dabrowski, Phys. Rev. D 71, 103505 (2005). arXiv:gr-qc/0410033

62. L. Fernandez-Jambrina, R. Lazkoz, Phys. Rev. D 70, 121503 (2004). arXiv:gr-qc/0410124

63. H. Stefancic, Phys. Rev. D 71, 084024 (2005). arXiv:astro-ph/0411630

64. M.P. Dabrowski, Phys. Lett. B 625, 184 (2005). arXiv:gr-qc/0505069

65. C. Cattoen, M. Visser, Class. Quant. Grav. 22, 4913 (2005). arXiv:gr-qc/0508045

66. P. Tretyakov, A. Toporensky, Y. Shtanov, V. Sahni, Class. Quant. Grav. 23, 3259 (2006). arXiv:gr-qc/0510104

67. M. Sami, P. Singh, S. Tsujikawa, Phys. Rev. D 74, 043514 (2006). arXiv:gr-qc/0605113

68. L. Fernandez-Jambrina, R. Lazkoz, Phys. Rev. D 74, 064030 (2006). arXiv:gr-qc/0607073

69. M. Bouhmadi-Lopez, P.F. Gonzalez-Diaz, P. Martin-Moruno, Phys. Lett. B 659, 1 (2008). arXiv:gr-qc/0612135

70. A.V. Yurov, A.V. Astashenok, P.F. Gonzalez-Diaz, Grav. Cosmol. 14, 205 (2008). arXiv:0705.4108 [astro-ph]

71. M. Bouhmadi-Lopez, P.F. Gonzalez-Diaz, P. Martin-Moruno, Int. J. Mod. Phys. D 17, 2269 (2008). arXiv:0707.2390 [gr-qc]

72. L. Fernandez-Jambrina, R. Lazkoz, Phys. Lett. B 670, 254 (2009). arXiv:0805.2284 [gr-qc]

73. I.H. Brevik, O. Gorbunova, Eur. Phys. J. C 56, 425 (2008) arXiv:0806.1399 [gr-qc]

74. J.D. Barrow, S.Z.W. Lip, Phys. Rev. D 80, 043518 (2009). arXiv:0901.1626 [gr-qc]

75. M. Bouhmadi-Lopez, C. Kiefer, B. Sandhofer, P.V. Moniz, Phys. Rev. D 79, 124035 (2009). arXiv:0905.2421 [gr-qc]

76. M. Bouhmadi-Lopez, Y. Tavakoli, P.V. Moniz, JCAP 1004, 016 (2010). arXiv:0911.1428 [gr-qc]

77. K. Bamba, S.D. Odintsov, L. Sebastiani, S. Zerbini, Eur. Phys. J. C 67, 295 (2010). arXiv:0911.4390 [hep-th]

78. J.D. Barrow, S. Cotsakis, A. Tsokaros, Class. Quant. Grav. 27, 165017 (2010). arXiv:1004.2681 [gr-qc] 
79. J.D. Barrow, A.B. Batista, J.C. Fabris, M.J.S. Houndjo, G. Dito, Phys. Rev. D 84, 123518 (2011). arXiv:1110.1321 [gr-qc]

80. Z. Keresztes, L.A. Gergely, A.Y. Kamenshchik, V. Gorini, D. Polarski, Phys. Rev. D 88, 023535 (2013). arXiv:1304.6355 [gr$\mathrm{qc}]$
81. S. Capozziello, S. Nojiri, S.D. Odintsov, Phys. Lett. B 634, 93 (2006). arXiv:hep-th/0512118 